\title{
Using monitoring data of surface soil to predict whole crop-root zone soil water content with PSO-LSSVM, GRNN and WNN
}

\author{
Zhao Lixi • Shui Pengbo • Jiang Fang • Qiu Hengqing • \\ Ren Shumei $\cdot$ Li Yunkai $\cdot$ Zhang Yu
}

Received: 5 November 2012 / Accepted: 23 August 2013 / Published online: 17 September 2013

(C) Springer-Verlag Berlin Heidelberg 2013

\begin{abstract}
Drought is a significant disaster in Beijing and it is important to find a method to assess the drought condition. First, this paper collected data of 85 soil monitoring stations in Beijing, such as soil dry bulk densities, saturated water contents, field capacities. Then, spatial variability characteristics of soil physics parameters were investigated by GIS and other three factors, $10 \mathrm{~cm}$ soil moisture content, organic matter and saturated water content which notably influenced soil moisture were extracted by Principal Component Analysis (PCA). Furthermore, four different nonlinear methods were put forward to predict crop-root zone soil water. 15555 single daily data from 2011 were used in parameters determination, while 15470 double daily data were used to test. The result showed that the Least Square Support Vector Machine coupling Particle Swarm Optimization Algorithm (PSO-LSSVM) $\left(R^{2}=0\right.$. $875)$ did better than BP Neural Network $\left(R^{2}=0.840\right)$, Generalized Regression Neural Network (GRNN) $\left(R^{2}=0.850\right)$ and Wavelet Neural Network (WNN) $\left(R^{2}=0.853\right)$. As so the POS-LSSVM method was used to evaluate the drought conditions from October 2010 to March 2011 of Beijing, and the result showed that from October 2010 to January 2011, the drought conditions were getting increasingly worse while later relieved from January 2011 to March 2011.
\end{abstract}

Communicated by: H. A. Babaie

Z. Lixi $\cdot$ J. Fang $\cdot$ Q. Hengqing $\cdot$ R. Shumei $\cdot$ L. Yunkai $(\bowtie) \cdot Z$. Yu Water Conservancy and Civil Engineering College, China Agricultural University, 100083 Beijing, Peoples Republic of China e-mail: liyunkai@126.com

\section{S. Pengbo}

Beijing Water Authority, 100073 Beijing, Peoples Republic of China

L. Yunkai

State Key Laboratory of Urban and Regional Ecology, Research Centre for Eco-Environmental Sciences, Chinese Academy of Sciences, Beijing 100085, Peoples Republic of China
Keywords Drought condition · Crop-root zone soil water · PSO-LSSVM · PCA

\section{Introduction}

Drought circumvents much the development of agriculture, especially for China, which is a large agricultural country. (Sun 2004; Li 2001) Located in mid-arid and arid area, Beijing is likely to be affected by drought, in addition with the other 12 counties listed to be drought-prone areas in China. It is important to find some methods to monitor the drought condition and provided proper predictions for the irrigation guiding as well as optimization of water resources (Wang et al. 2012; Mozny et al. 2012). The drought condition usually measures by average soil moisture in the entire soil profile, which is difficult to be measured. How to use the surface soil moisture (like $0 \mathrm{~cm} \sim 10 \mathrm{~cm}$ soil moisture) to predict the average soil moisture is an economic way to predict drought condition. To measure the soil moisture, the soil moisture sensor was considered to be the most convenient and quick equipment. In order to monitor a vast area, more sensors were needed, different depths needed to be measured, generating a significant amount of redundant data and cost. Thus it's urgent to figure out how to reduce the cost and increase precision with the surface soil moisture measured.

Based on the collected data such as soil dry bulk densities, saturated water contents, $\mathrm{pH}$ values, organic matters and field capacities of 85 soil monitoring stations in Beijing, the paper analyzed factors that influenced crop-root zone soil water notably via principal component analysis (PCA). Meanwhile, our different nonlinear methods, PSO-LSSVM, BP neural network, GRNN and WNN, were put forward to predict crops root zone soil water (average soil moisture). Finally, drought conditions from October 2010 to March 2011 of Beijing were evaluated by PSO-LSSVM. 


\section{Methods and materials}

The condition of drought monitors in Beijing

There are 85 soil monitoring stations in Beijing (Fig. 1), which supported our studies significantly. The soil types, soil dry bulk densities, saturated water contents, $\mathrm{pH}$ values, organic matters and field capacities were collected and competed during the 4 months in 2011. To measure the average soil moisture, we put TSC series intelligent soil moisture measuring instrument in three layers of $0 \mathrm{~cm} \sim 10 \mathrm{~cm}, 10 \mathrm{~cm} \sim 30 \mathrm{~cm}$, $30 \mathrm{~cm} \sim 50 \mathrm{~cm}$. One sensor was inserted vertically at the earth surface, whose prob was $10 \mathrm{~cm}$. And two sensors were buried horizontally under the earth surface of $20 \mathrm{~cm}$ and $40 \mathrm{~cm}$ to measure the soil moisture of $10 \mathrm{~cm} \sim 30 \mathrm{~cm}$ and $30 \mathrm{~cm} \sim 50 \mathrm{~cm}$. The saturated water contents and the field capacities were calculated according to the Water Standard of the People's Republic of China of Soil Moisture Monitoring Specification (SL364-2006). And the average soil moisture is calculated by averaging the soil moisture in $0 \mathrm{~cm} \sim 10 \mathrm{~cm}, 10 \mathrm{~cm} \sim 30 \mathrm{~cm}$, $30 \mathrm{~cm} \sim 50 \mathrm{~cm}$.

\section{PSO-LSSVM}

SVM was raised by Vapnik and his fellows in 1995 (Deng and Tian 2004) as a new mechanic learning method and applies well in many areas (Li et al. 2009). Least Square Support Vector Machine (LSSVM) is developed from SVM but being computationally more efficient. Its details can be found in Suykens et al. (2002). The principle of LSSVM is:

Considering a given training set, $i=1,2, \ldots n$, where $x_{i} \in R^{d}$ represents a $i$-dimensional input vector and $y_{j} \in R$, which is a scalar measured data, represents the system output. The form of this function is defined as

$y(x)=w^{T} \varphi\left(x_{i}\right)+b$

Where, $w$ is the weight vector, $b$ is the bias term and $\varphi(\cdot)$ is a nonlinear function that maps the input space into a higher dimension feature space. Finding the optimal parameters that minimize the prediction error of the regression model, the following optimization problem with equality constraints:

$\min J(w, e)=\frac{1}{2} w^{T} w+\frac{\gamma}{2} \sum_{i=1}^{n} e_{i}^{2}$

Subject to:

$y_{i}=w^{T} \varphi\left(x_{i}\right)+b+e_{i}, \quad i=1,2, \cdots, n$

Where $e_{i}$ is the random error and $\gamma \in R^{+}$is the regularization parameter determines the trade-off of minimizing the training errors and the model complexity.
To solve the optimization problem (Eq. 3), the Lagrange function is constructed as the following:

$L(w, b, e, \alpha)=\frac{1}{2} w^{T} w+\frac{\gamma}{2} \sum_{i=1}^{n} e_{i}^{2}-\sum_{i=1}^{n} \alpha_{i}\left\{w^{T} \varphi\left(x_{i}\right)+b-1+e_{i}-y_{i}\right\}$

The solution to the constrained optimization problem is determined by the saddle point of the Lagrange function, which has to be minimized with respect to $w, b, e_{i}$, and $\alpha_{i}$ (Fletcher 1987). And the solution of Eq. (4) can be obtained:

$$
\begin{aligned}
& \frac{\partial L}{\partial w}=0 \Rightarrow w=\sum_{i=1}^{n} \alpha_{i} \varphi\left(x_{i}\right) \\
& \frac{\partial L}{\partial b}=0 \Rightarrow \sum_{i=1}^{n} \alpha_{i}=0 \\
& \frac{\partial L}{\partial e_{i}}=0 \Rightarrow \alpha_{i}=\gamma e_{i} \\
& \frac{\partial L}{\partial \alpha_{i}}=w^{T} \varphi\left(x_{i}\right)+b+e_{i}-y_{i}=0 \quad(i=1,2, \cdots n)
\end{aligned}
$$

In the form of matrix, those four equations can be expressed as:

$$
\left[\begin{array}{cc}
0 & \Theta^{T} \\
\Theta & \Omega+\gamma^{-1} I
\end{array}\right]\left[\begin{array}{l}
b \\
\alpha
\end{array}\right]=\left[\begin{array}{l}
0 \\
y
\end{array}\right]
$$

Where, $y=\left[y_{1}, y_{2}, \cdots, y_{n}\right]^{T}, \Theta=[1,1, \cdots, 1]^{T}, \alpha=\left[\alpha_{1}, \alpha_{2}\right.$, $\left.\cdots, \alpha_{n}\right]^{T}, \Omega$ is a square matrix, and $\boldsymbol{I}$ is an identity matrix. The elements $\Omega_{i j}=\kappa\left(x_{i}, x_{j}\right), i, j=1, \ldots, n$. Finally, the estimated values of $\boldsymbol{\alpha}$ and $b$ can be obtained by solving the linear system of Eq. (6), and the resulting LSSVM model can be expressed as:

$f(x)=\sum_{i=1}^{n} \alpha_{i} \kappa\left(x_{i}, x\right)+b$

Where, $\kappa\left(x_{i}, x\right)$ is the kernel function. In comparison with other kernel functions, the Radial Basis Function (RBF) is better compact supported and able to reduce the computational complexity of LSSVM's training process. (Smola 1998) So, the RBF kernel was selected as the kernel function:

$\kappa\left(x_{i}, x\right)=\exp \left(-\frac{1}{2 \sigma^{2}}\left\|x-x^{i}\right\|^{2}\right)$

Where, $\sigma$ is the kernel width parameter.

Based on RBF kernel, the model of the LSSVM can be expressed as:

$y(x)=\sum_{i=1}^{n} \alpha_{i} \exp \left(-\frac{1}{2 \sigma^{2}}\left\|x-x^{i}\right\|^{2}\right)+b$ 
Fig. 1 Monitoring stations of soil moisture contents in Beijing

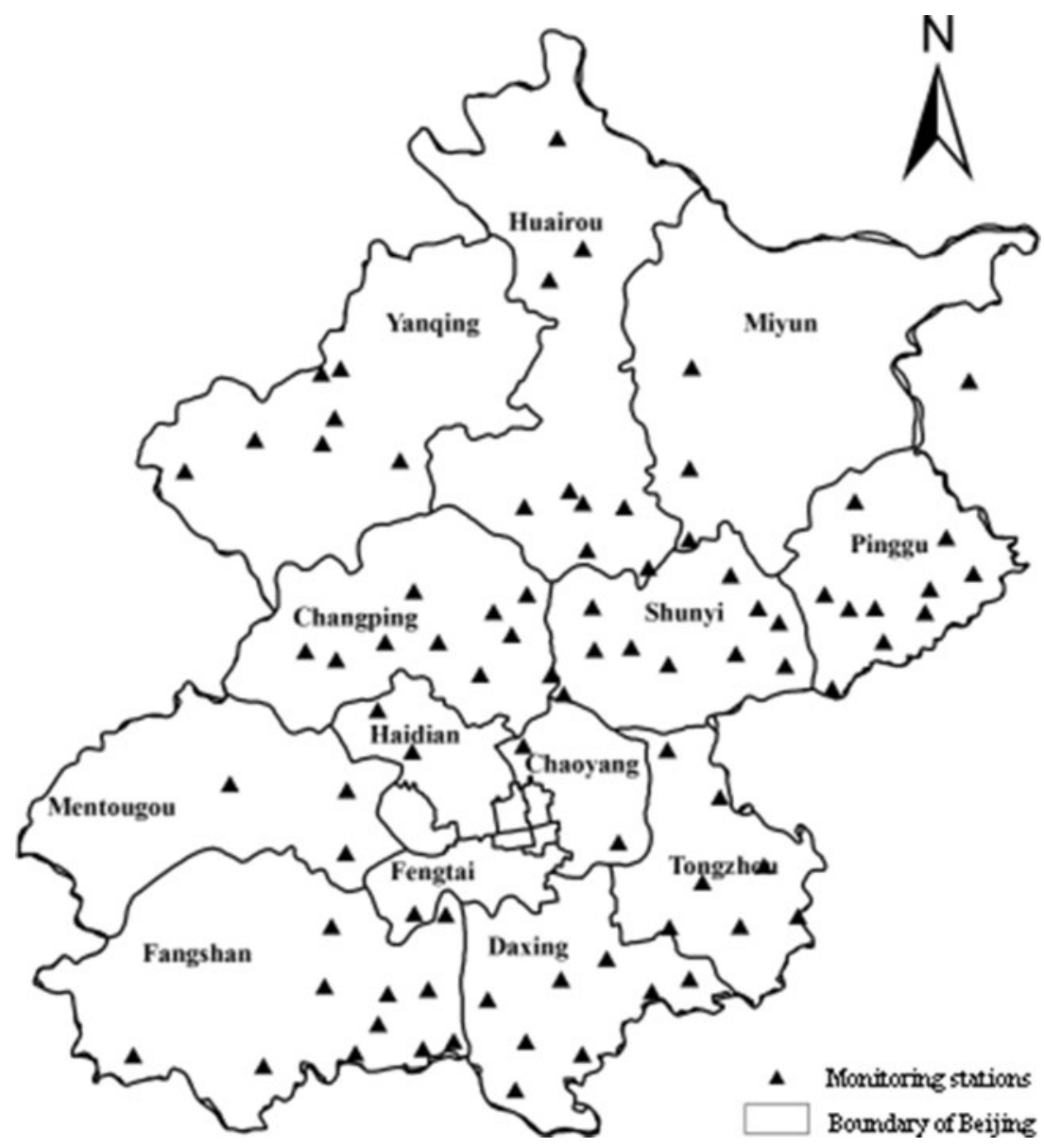

PSO was a bionic optimization algorithm proposed by Kennedy and Eberhart. (Kennedy and Eberhart 1995) At the outset of the algorithm, PSO made particle swarm initialized as random particles. Then the particle tailed two extremes to update itself finding the best solutions for each iteration. After the two best solutions are found, according to Eqs. (10) and (11) the particle renewed the owned speed and position.

The information of particle $i$ can be presented by a $d$ dimensional matrix, the position could be presented as $Y_{i}=$ $\left(y_{i 1}, y_{i 2}, \ldots, y_{i d}\right)^{T}$, the speed was $V=\left(\nu_{i 1}, \nu_{i 2}, \ldots, \nu_{i d}\right)^{T}$, other vectors were similar, then,

$$
\begin{aligned}
\nu_{i d}^{k+1}= & w \times v_{i d}^{k}+c_{1} \times \operatorname{rand}_{1}^{k} \times\left(\text { pbest }_{i d}^{k}-x_{i d}^{k}\right) \\
& +c_{2} \times \text { rand }_{2}^{k} \times\left(\text { gbest }_{i d}^{k}-x_{i d}^{k}\right) \\
y_{i d}^{k+1}= & y_{i d}^{k}+v_{i d}^{k+1}
\end{aligned}
$$

Where, $v_{i d}{ }^{k}$ is particle $i$ in the $k$-th iteration of the ddimensional speed; pbest $_{d}$ is the individual extreme point position where particle $i$ locates in the $d$-dimensional; gbest ${ }_{d}$ is the overall situation extreme point position where the entire particle group locates in the d-dimensional; $c_{1}, c_{2}$ are accelerating coefficients; $x_{i d}{ }^{k}$ is particle $i$ in the $k$-th iteration, within $d$-dimension of the current location; $W$ is the initial weight value of inertia.

The realization of $P S O-L S S V M$ is equivalent to making the LSSVM model's structure and the forecast algorithm inserted into the step of PSO computing adaptation values. The concrete algorithm flow is:

(1) If a group of $g, s$ is seeking by a particle of PSO algorithm module, it will transfer to (2);

(2) Constructing $L S S V M$ by the group of parameters, carrying on the data five pays the fork confirmation proportionately, and taking the standard value as the sufficiency value back to the PSO arithmetic module, then transfer to (3);

(3) If all PSO algorithm's particles have been taken step (2) for sufficiency values, transfer to (4), otherwise return to (1);

(4) Renewing every particle of PSO algorithm positional information, speed information, local extremum and so on, then transfer to (5);

(5) The PSO algorithm module judges whether to achieve the withdrawal condition. If the withdrawal condition is met, it will withdraw and return the optimal-adaptive value, otherwise return to (1). 


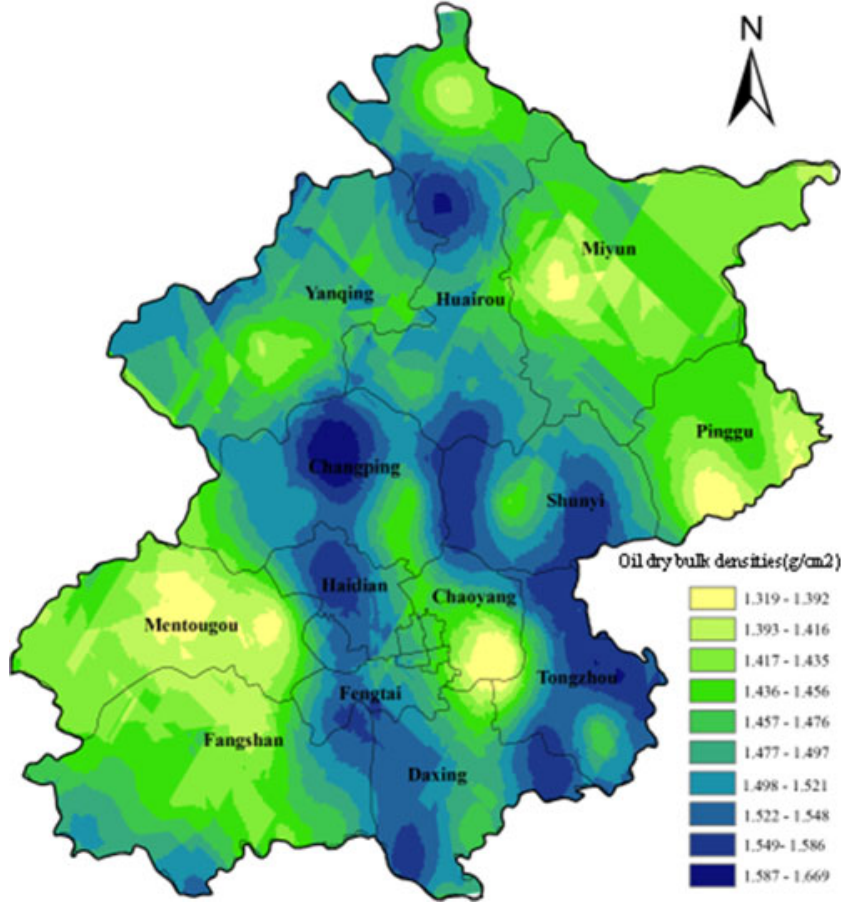

(a) Soil dry bulk densities

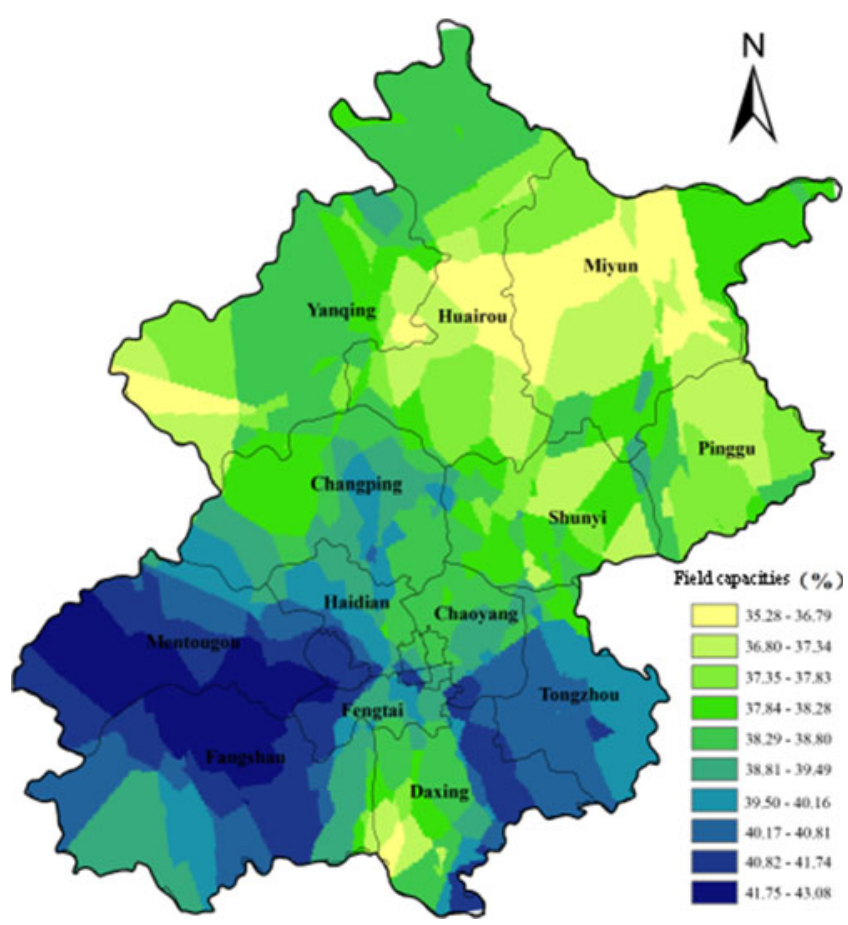

(c) Field capacities

Fig. 2 The spatial variations of soil physical characteristics in Beijing. The soil dry bulk densities of Beijing (a) suggested that it kept increasing from Chaoyang, Miyun, Mentougou to the extend. The maximum was $1.6 \mathrm{~g} / \mathrm{cm}^{3}$ presented in Changping, Shunyi and Tongzhou. Saturated water contents (b) showed that it kept rising from Daxing and Chaoyang to other areas. Especially parts of Mengtougou, Fangshan and Pinggu,

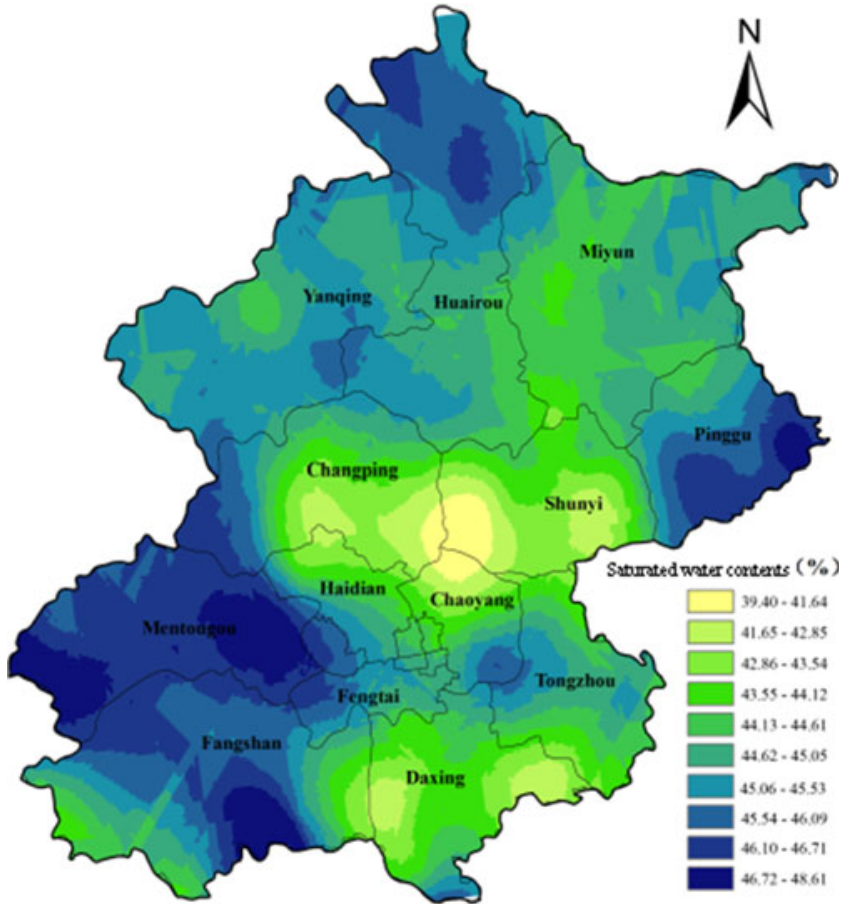

(b) Saturated water contents

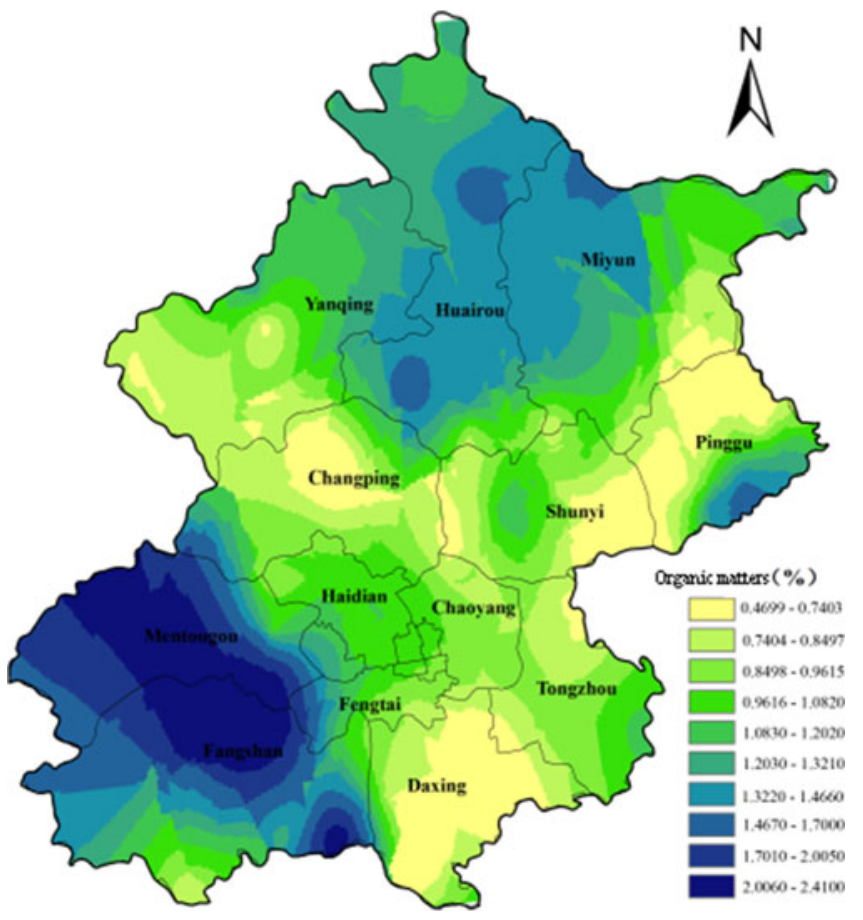

(d) Organic matters

they have values higher than $45.5 \%$. Field capacities showed on (c) suggested that it declined from the south to the north gradually. Mentougou and Tangshan have higher values, which is $41.8 \%-43.8 \%$, while Miyun held the minimum of $35.5 \%-36.8 \%$. Organic matters (d) showed that the value of Mengtougou was higher than that of Daxing, Shunyi, Changping and Pinggu 
Table 1 The rate of contribution of factors by PCA

\begin{tabular}{|c|c|c|c|c|c|c|}
\hline Factors & $10 \mathrm{~cm}$ soil moisture contents & Organic matters & Saturated water contents & Field capacities & Soil dry bulk densities & $\mathrm{pH}$ values \\
\hline Percentages & $78.01 \%$ & $14.59 \%$ & $7.07 \%$ & $0.31 \%$ & $0.01 \%$ & $0.01 \%$ \\
\hline
\end{tabular}

\section{PCA}

PCA is a useful statistical technique that applies in multiple fields such as face recognition and image compression. It is also a common technique for finding patterns in data with high dimensions. It identifies patterns in data and expresses them while highlighting the similarities and differences. The steps of PCA are shown as the following (Ian and Eibe 2005):

Considering a given matrix $X=\left[X_{1}, X_{2}, \ldots X_{n}\right], n \in N^{+}$, and $X_{n}=\left[x_{1 n}, x_{2 n}, \ldots x_{i n}\right]^{T}, i \in N^{+}$,

$m=\frac{1}{i} \sum_{j=1}^{i} x_{i}$

Then, $Y_{n}=\left[x_{1 n}-m, x_{2 n}-m, \ldots x_{i n}-m\right]^{T}$. Its covariance matrix could be calculated:

$S=\frac{Y Y^{T}}{i-1}$

Where $S$ was transformed by eigen decomposition, and the eigenvalues $e_{n}$ and eigenvectors $\lambda_{n}$ were extracted. Make $E$ $=\left[e_{1}, e_{2}, \ldots, e_{n}\right]$, then,

$S E=\left[e_{1}, e_{2}, \cdots e_{i}\right]\left[\begin{array}{ccc}\lambda_{1} & \cdots & 0 \\ \vdots & \ddots & \vdots \\ 0 & \cdots & \lambda_{i}\end{array}\right]=E D$

Where, $D=\left[\begin{array}{ccc}\lambda_{1} & \cdots & 0 \\ \vdots & \ddots & \vdots \\ 0 & \cdots & \lambda_{i}\end{array}\right]$

Finally, the percentage of $X_{n}$ could be got though the following equation:

$p_{k}=\frac{\lambda_{k}}{\sum_{j=1}^{n} \lambda_{j}} \times 100 \%$

Drought assessment

To relief the plant from drought, objective and precise assessment of drought is necessary. For the variation between climates, hydrology and economies, different methods could be chosen to assess drought in different areas, such as, the relative soil moistures, percentage of precipitation anomalies, consecutive days without rains, irrigation water deficiency ratios and consecutive days without water in rice-field. According to the Standard of Drought Classification of the Water Resources in China (SL424-2008), soil moisture is an important indicator for drought assessment in agriculture. Considering that there are large areas of vegetation planted in Beijing, the paper used 0-40 cm relative soil moistures to make the assessment. The calculation formula is shown as follows:

$W=\frac{\theta}{F_{C}} \times 100 \%$

Where, $W$ is relative soil moisture (\%); $\theta$ is the average weight of soil water content (\%); $F_{C}$ is field capacity (\%). If $50<\mathrm{W} \leq 60$, it is mild drought; if $40<\mathrm{W} \leq 50$, it is moderate drought; If $30<\mathrm{W} \leq 40$, it is severe drought; If $W \leq 30$, it is extraordinary drought.

\section{Conclusions and analyses}

The spatial variations and features extraction of soil physical characteristics

Space interpolation method of GIS was used to analyze the spatial variations of soil dry bulk densities, saturated water contents, organic matters and field capacities. The results are shown in Fig. 2.

PCA was used to analyze the $10 \mathrm{~cm}$ soil moisture contents, soil dry bulk densities, saturated water contents, organic matters and field capacities. The results were showed in Table 1.

The $10 \mathrm{~cm}$ soil moisture contents had a much tighter relationship with the average soil water contents. Organic matters and saturated water contents took the second places. However, field capacities, soil dry bulk densities as well as $\mathrm{pH}$ values were minor. The paper preserved $95 \%$ of those data, thus $10 \mathrm{~cm}$ soil moisture contents, organic matters and saturated water contents were considered to be the main factors.

Model simulation and precision analyses

The paper adopted $0 \mathrm{~cm} \sim 10 \mathrm{~cm}$ soil moisture contents, organic matters and saturated water contents which were got by PCA, together with four different methods, PSO-LSSVM, BP network, GRNN and WNN for training and prediction. This paper collected daily data of 85 soil monitoring stations in Beijing in 2011. Hence, 15555 single daily data from 2011 


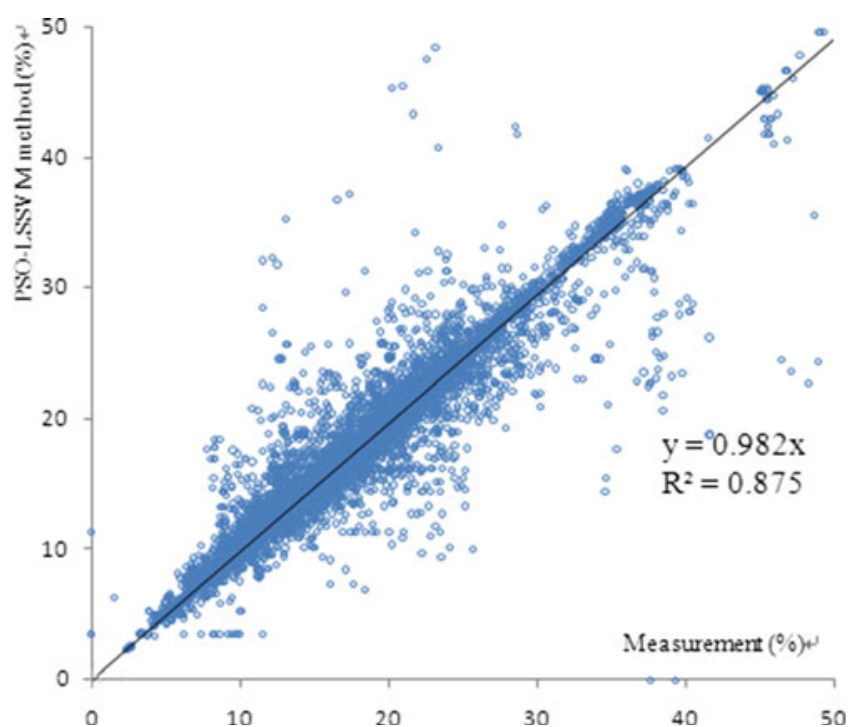

(a) PSO-LSSVM

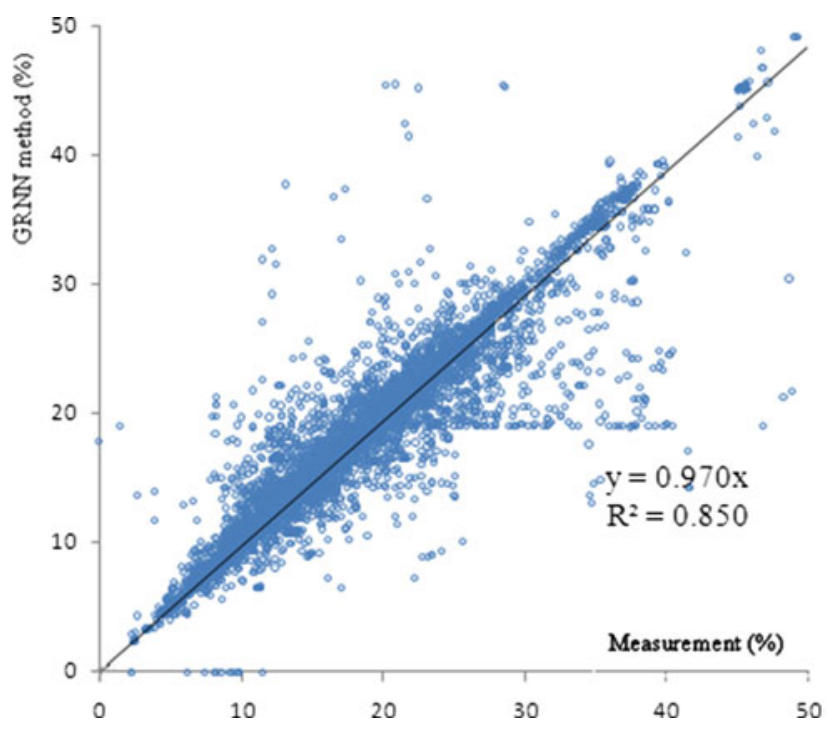

(c) GRNN

Fig. 3 The results prediction by four methods. Among those four methods, PSO-LSSVM got the highest accuracy; in addition, WNN did better than GRNN and BP; moreover, BP was worst in accuracy.

were used in parameters determination, while 15470 double daily data were used for testimony.

Unlike the SVM, LSSVM is more efficient in terms of computational complexity and difficulty of learning. The PSO algorithm's parameters were set as follows: the particle dimension $d$ is 3 , the number of the population $I$ is 20 , initial inertia weight $W$ is 0.9 , the maximum speed of the particle $v_{d \max }$ is 10 and the biggest position value of the particle $x_{d \max }$ is 100 . Finally, the most superior parameter $\gamma=35.6, \sigma=4.3$. According to these parameters, the PSO-LSSVM model was obtained by the

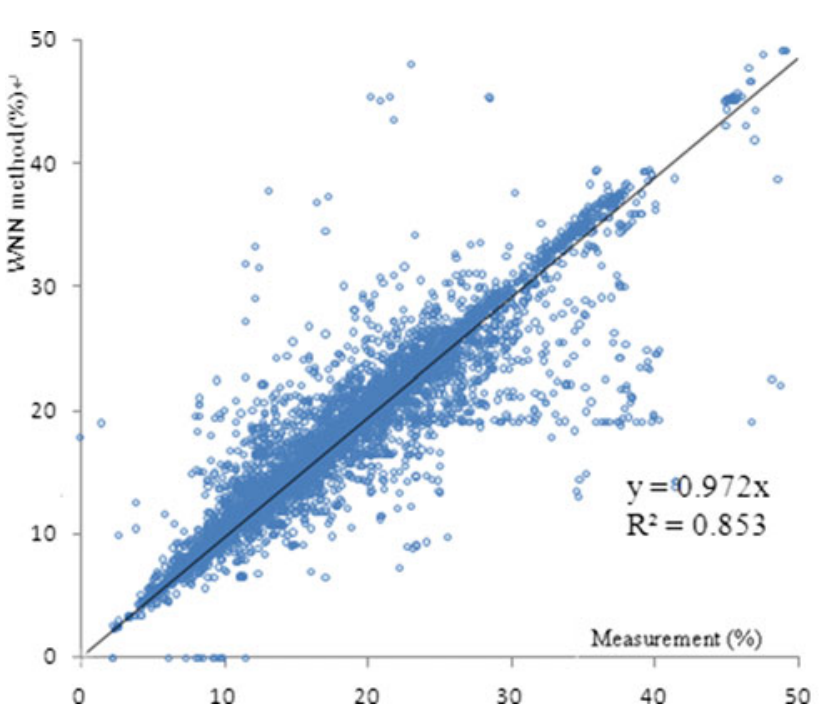

(b) $\mathrm{WNN}$

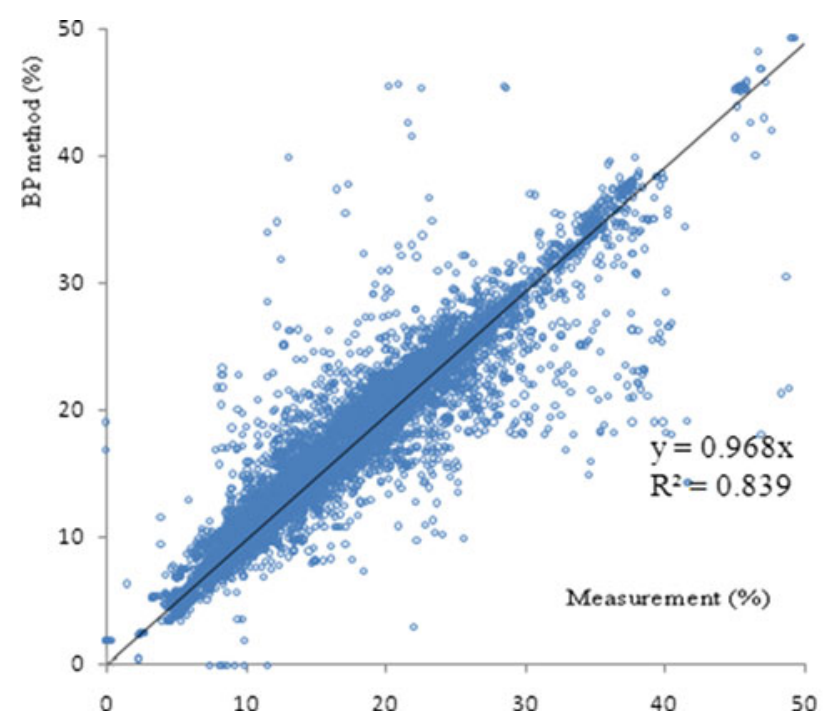

(d) BPnetwork

However, PSO took quite a long time for a great number of data; GRNN and BP take relatively less. But GRNN dominated a heavier memory virtual memory

training and forecasting results of testing data set shown in Fig. 3(a). The $\mathrm{x}$-axis is the measurement of average soil moisture in Beijing and the y-axis is the prediction of soil moisture in Beijing. The related coefficient $R=0.936$ and straight line equation $y=0.9823 x$ could fit the measurements and predictions. The paper also used other 3 different artificial neural networks to control. The powerful new type of artificial neural networks emerged as WNN was used. And there were five nods of input layer, one node of output layer and six numbers of hidden nodes, with 100 times of training. Based on these parameters, the results 


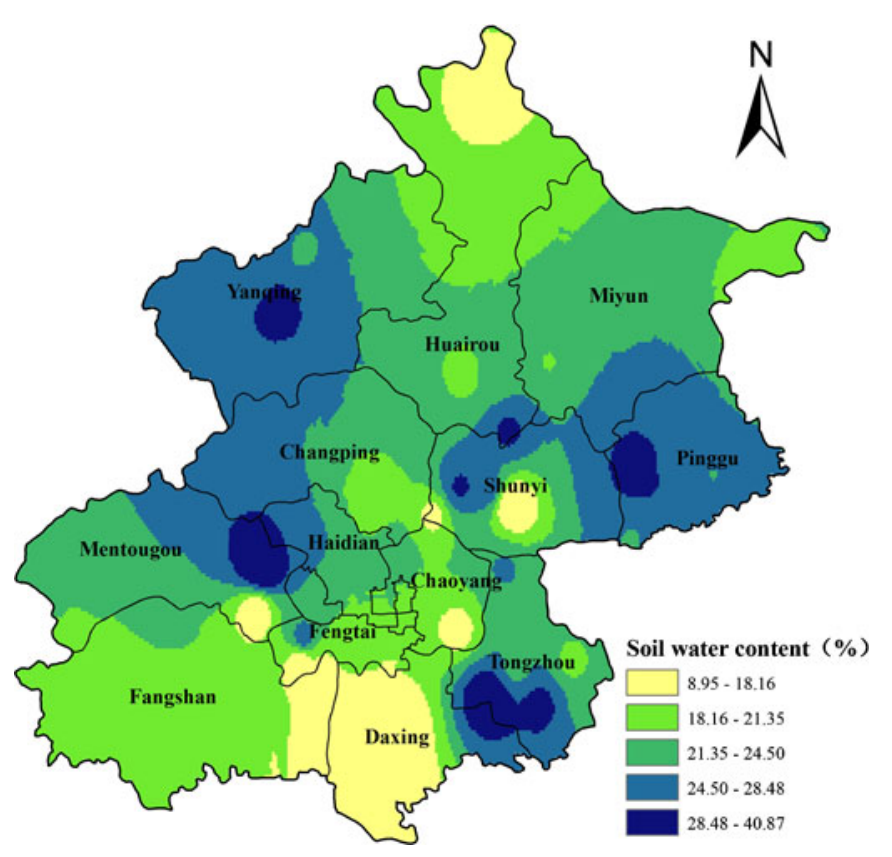

(a) The soil water content of October 2010

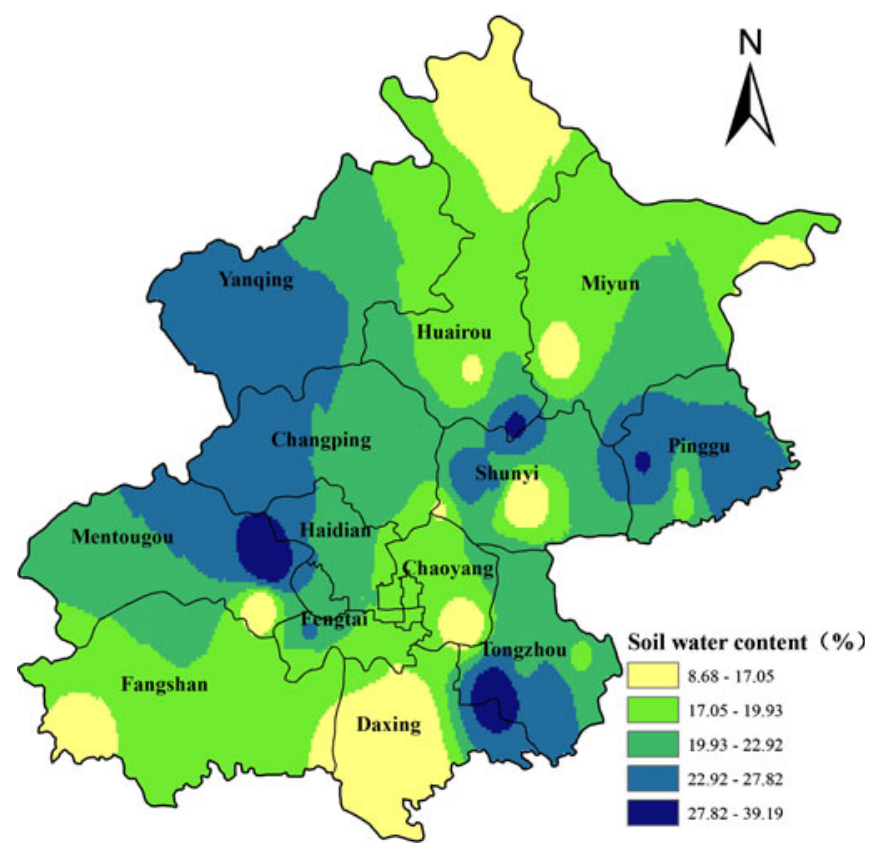

(c) The soil water content of November 2010

Fig. 4 The distribution of soil water content and drought from October 2010 to March 2011 of Beijing. Based on PSO-LSSVM, we took daily $0 \mathrm{~cm} \sim 10 \mathrm{~cm}$ soil moisture data to predict its soil moisture from October 2010 to March2011. Then, by aevraging every predcition daily data in each month, the monthly average soil water content was shown on (a), $\mathbf{c}$,

(Fig. 3(b)) are as follows, the related coefficient $R=0$. 923, and straight line equation $y=0.9723 x$ could fit the

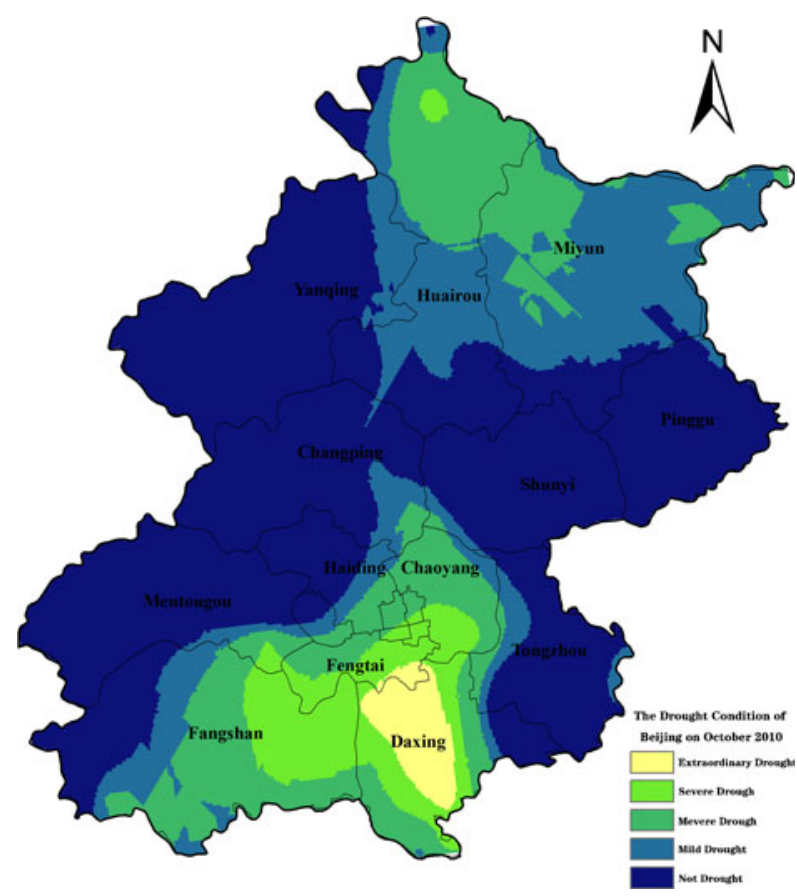

(b) The droughtcondition of October 2010

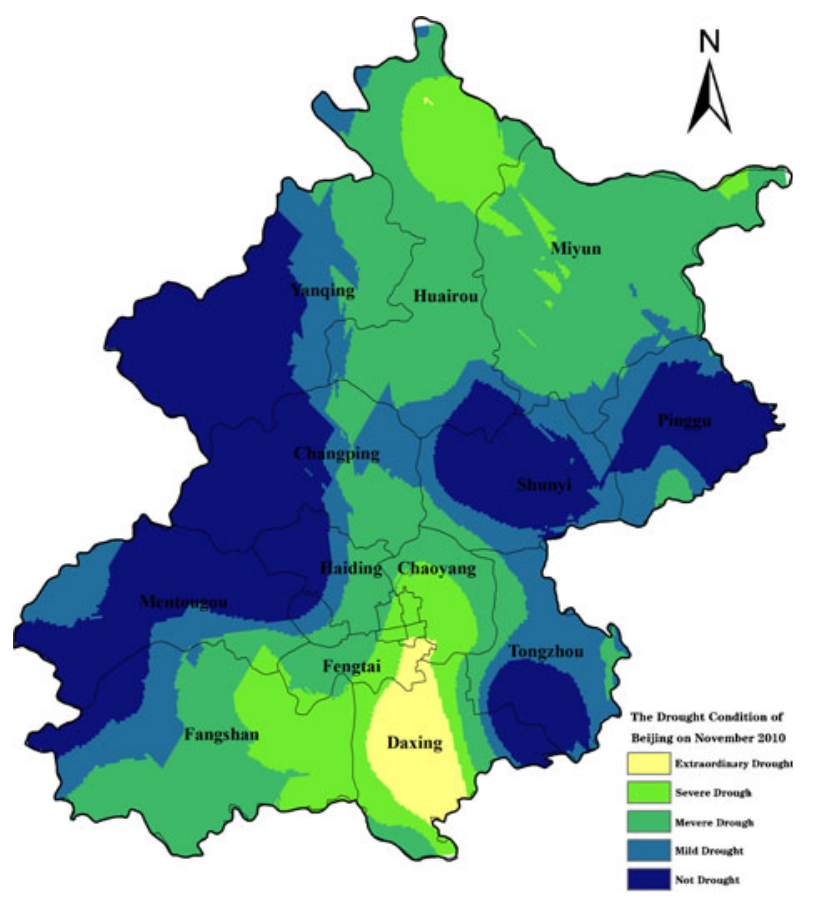

(d) The drought condition of November 2010

e, g, i and (k). In October 2010 and March 2011, the soil water content was separately $8.95 \% \sim 40.87 \%$ and $7.25 \% \sim 40.59 \%$. In November 2010 and February 2011, the soil water content was $8.68 \% \sim 39.19 \%$ and $4.84 \% \sim 37.08 \%$. In December 2010 and January 2011, the soil water content was $4.3 \% \sim 34.01 \%$ and $4.13 \% \sim 26.82 \%$

measurements and predictions; GRNN, which had a very strong nonlinear mapping capability and flexible network 


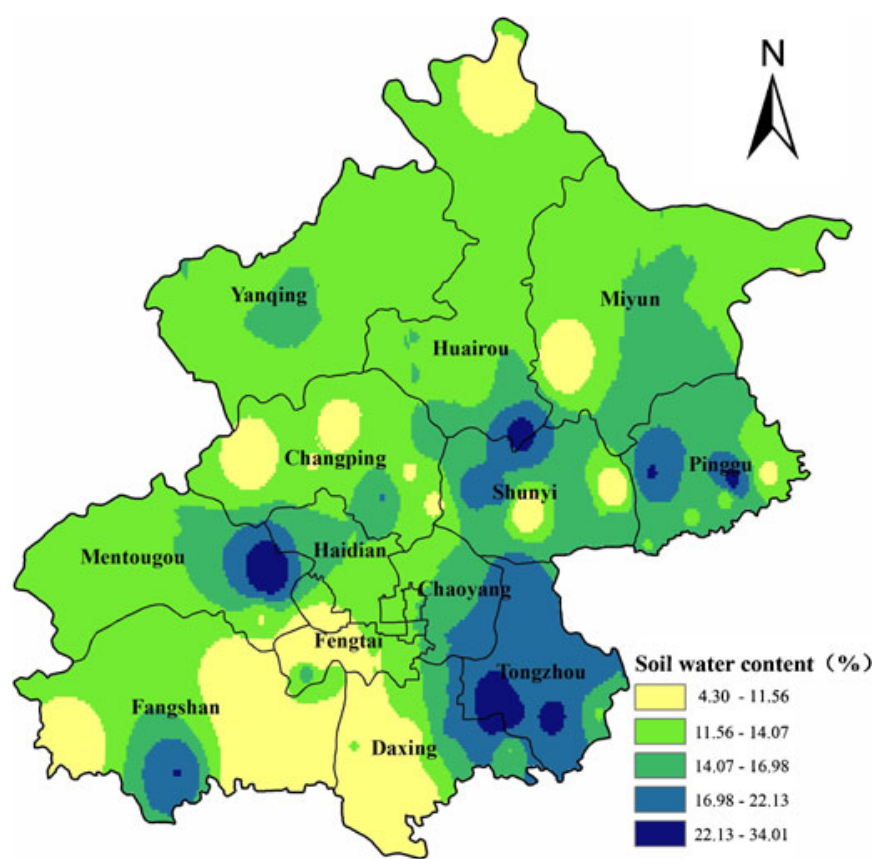

(e) The soil water content of December 2010

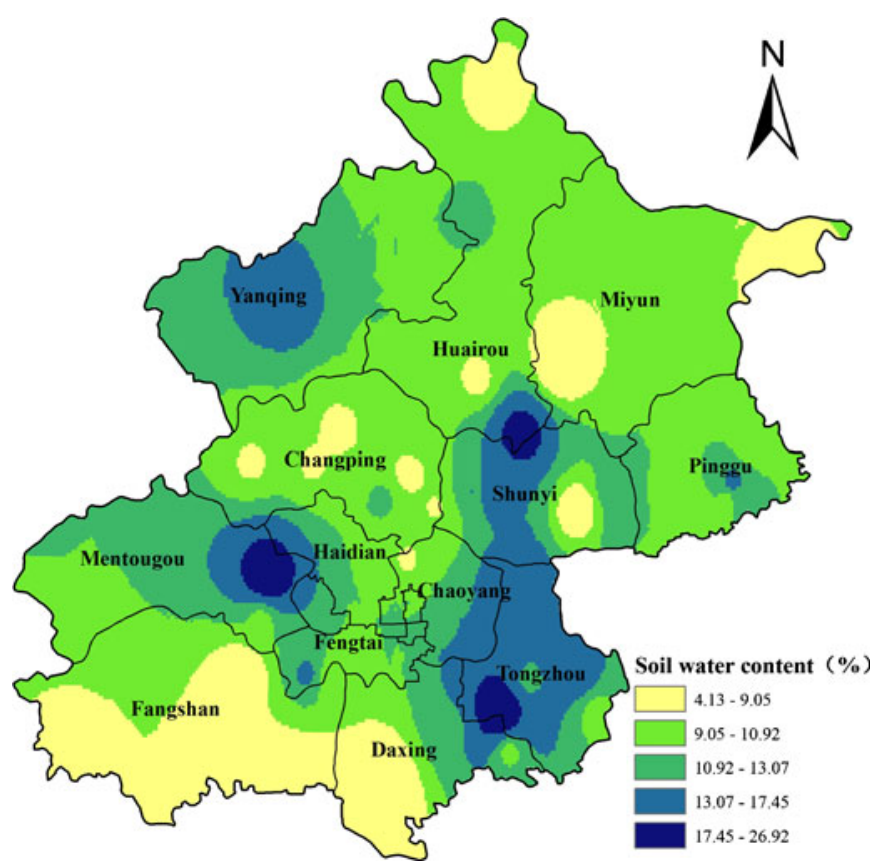

(g) The soil water content of January 2011

Fig. 4 (continued)

structure, showed (Fig. 3(c)) that the related coefficient $R=0.922$ and the straight line equation was $y=0.9693 x$; the result of BP network (Fig. 3(d)) were: the related coefficient $R=0.916$, and straight line equation was $y=$ $0.968 x$.

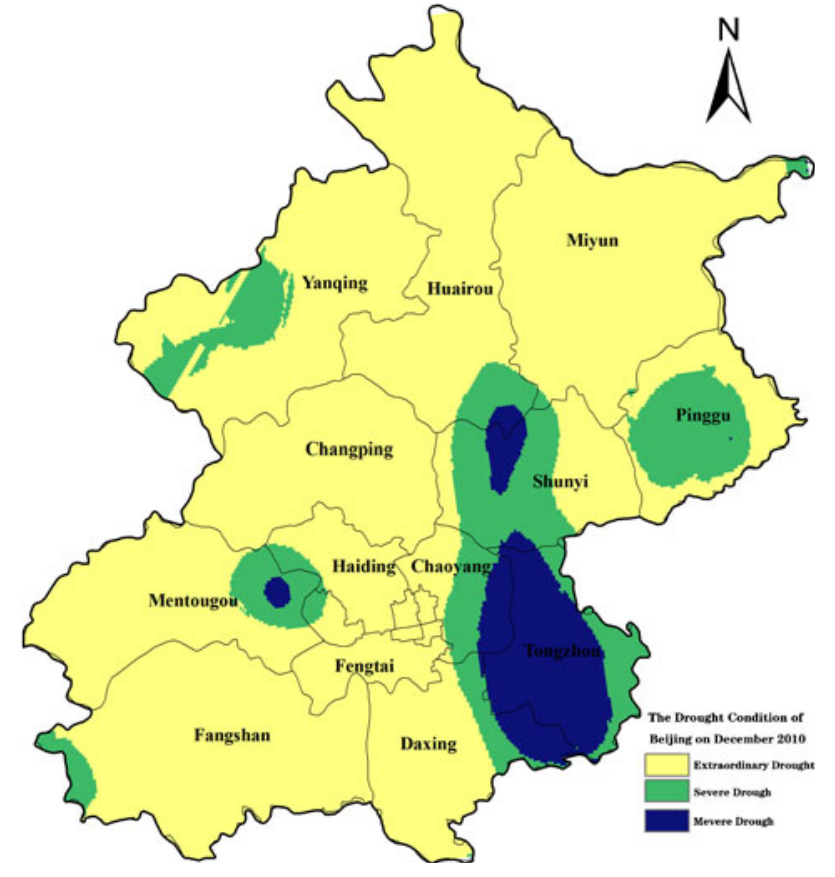

(f) The drought condition of December 2010

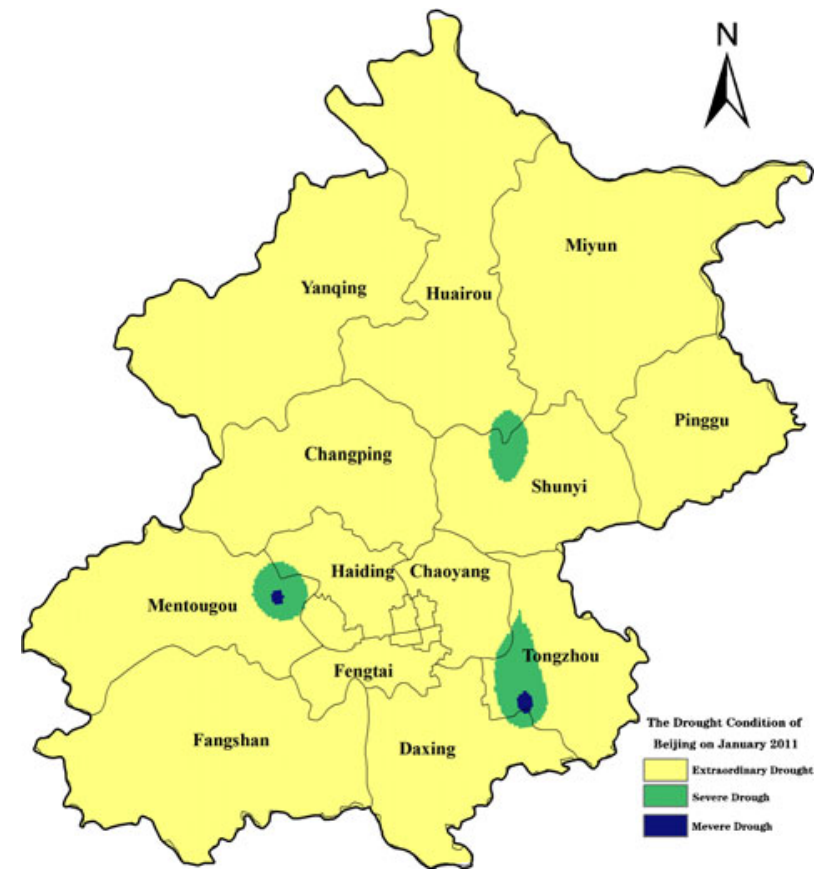

(h) The droughtcondition of January 2011

The assessment of drought

The POS-LSSVM method was used to evaluate the drought condition from October 2010 to March 2011 of Beijing. The results were shown on Fig. 4. 


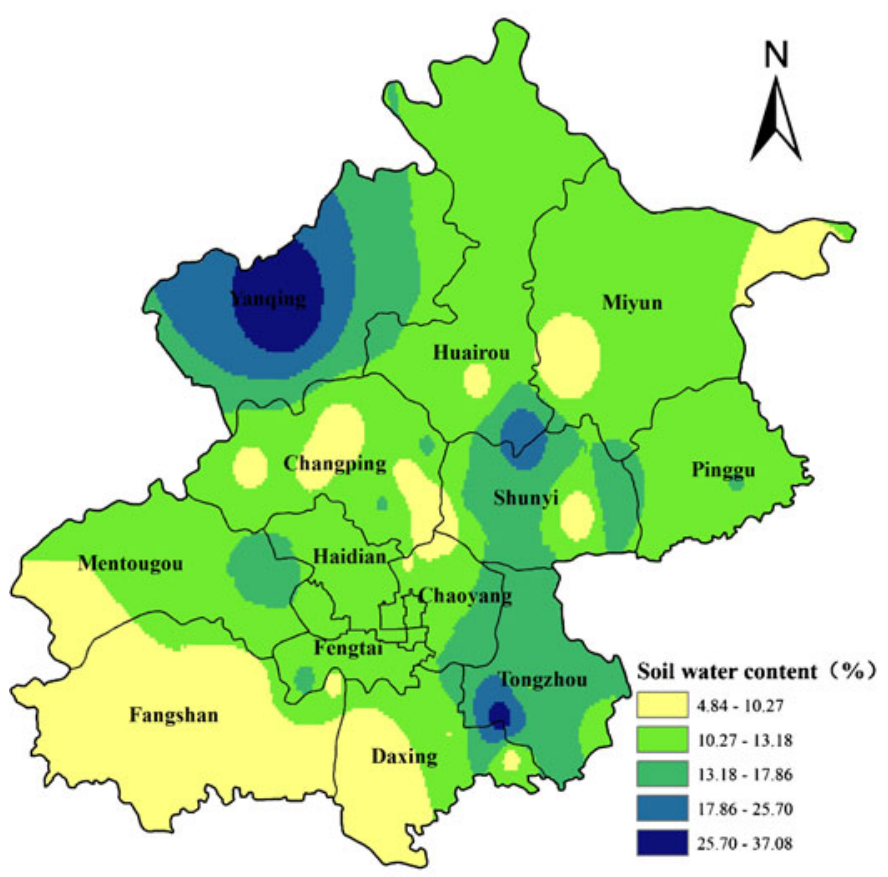

(i) The soil water content of February 2011

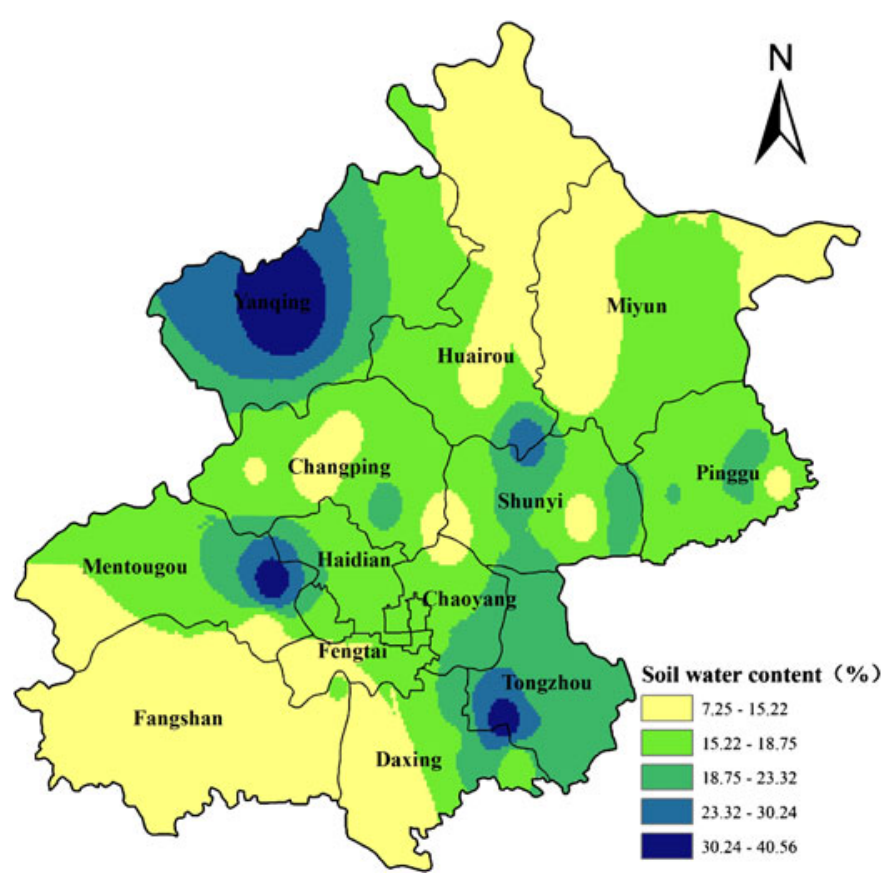

(k) The soil water content of March 2011

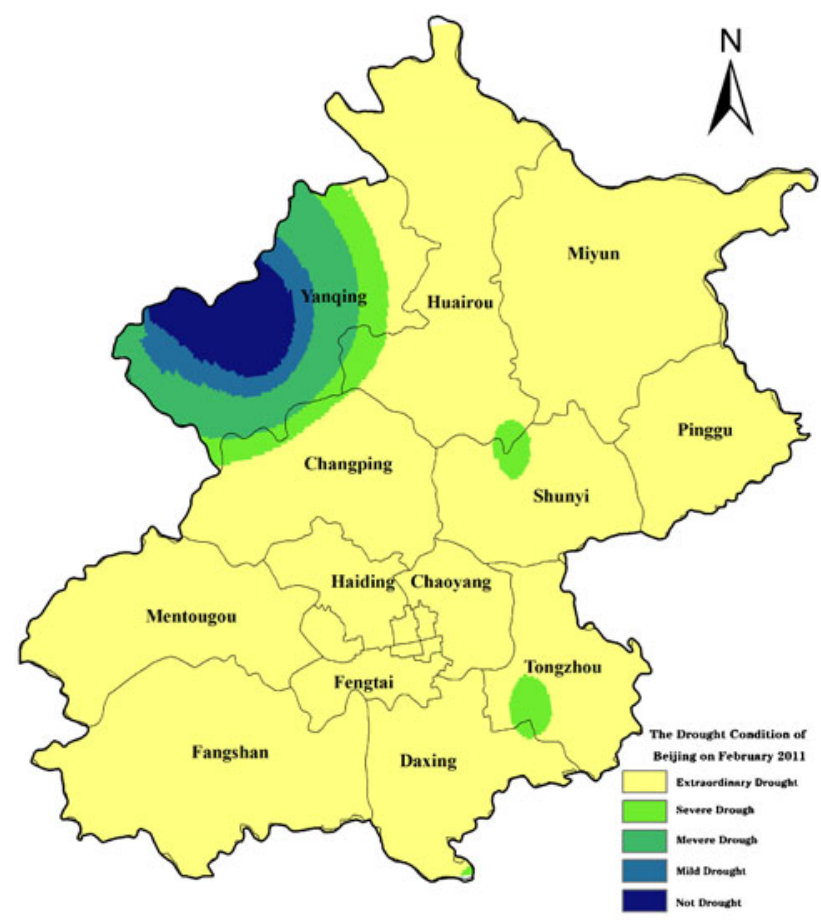

(j) The drought condition of February 2011

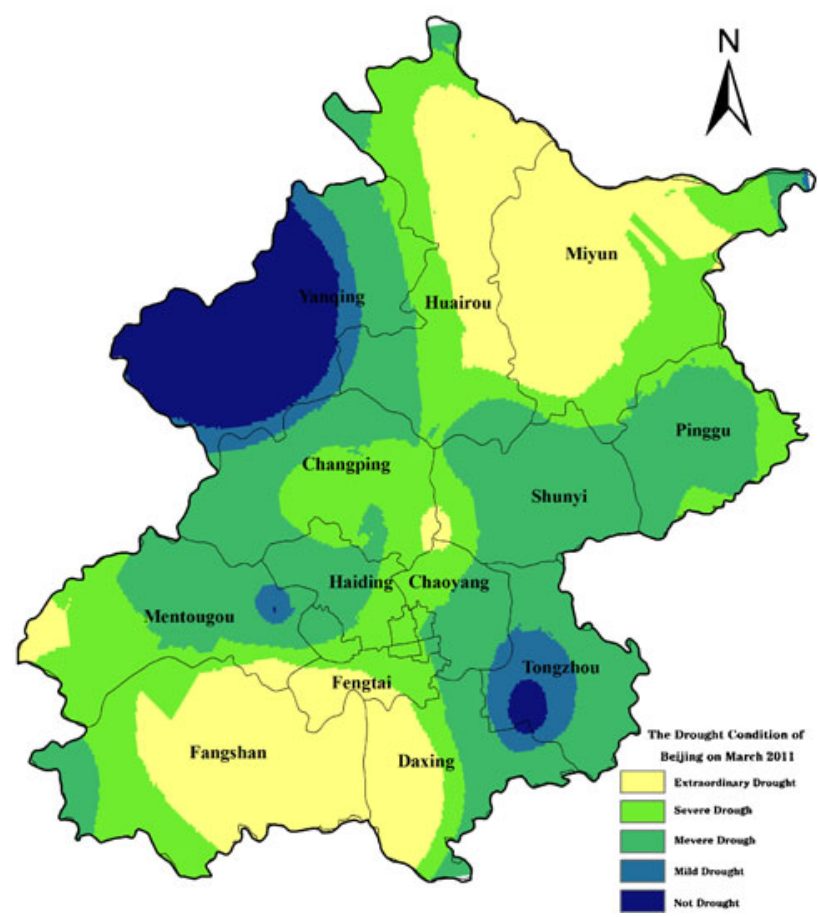

(I) The drought condition of march 2011

Fig. 4 (continued)

The drought conditions were shown on Fig. 4(b), (d), (f), (h), (j) and (l). From October 2010 to January 2011, the drought condition kept getting worse, especially on January
2011, most places were under extraordinary drought. In addition, the conditions got relived from January 2011 to March 2011. On October 2010, the drought was serious in 
southern and northern areas, especially in Daxing which was under extraordinary level. In the following month, western and eastern areas including Shunyi, Pinggu, Tongzhou and Yanqing were in mild drought. Others, however, were in moderate drought. On December 2010 and January 2011, the extraordinary-leveled drought hit most areas in Beijing. From February to March 2011, most droughts were relived and downed to moderate level or severe level.

\section{Conclusions}

Based on the analyses, three conclusions can be put forward.

1. Results from Principal Component Analysis (PCA) indicated that three factors, $10 \mathrm{~cm}$ soil moisture content, organic matter and saturated water content, have notably influenced on the soil water content.

2. PSO-LSSVM, WNN, GRNN and BP network could be used to predict soil moisture content and PSO-LSSVM $\left(R^{2}=0.875\right)$ did better than others.

3. The drought conditions, predicted by PSO-LSSVM, showed that the trend of drought was getting worse from October 2010 to January 2011, while reversed in March 2011.

\section{References}

Deng N, Tian Y (2004) New method in data mining-SVM. Science Press, Beijing

Fletcher R (1987) Practical methods of optimization. Wiley, Chichester

Ian HW, Eibe F (2005) Data mining: practical machine learning tools and techniques. Morgan Kaufmann

Kennedy J, Eberhart RC (1995) Particle swarm optimization[C]/Proc of IEEE International Conference on Neural Networks. IEEE Press, USA, pp 1942-1948

Li J (2001) Method and advances of drought monitoring by remote sensing. J China Hydrol. (04):15-17

Li Y, Tian Y, Ouyang Z, Wang L, Xu T, Yang P, Zhao H (2009) Analysis of soil erosion characteristics in small watersheds with particle swarm optimization, support vector machine, and artificial neuronal networks. Environ Earth Sci 60(7): $1559-1568$

Mozny M, Trnka M, Zalud Z, Hlavinka P, Nekovar J, Potop V, Virag M (2012) Use of a soil moisture network for drought monitoring in the Czech Republic. Theor Appl Climatol 107(1-2):99-111

Smola AJ (1998) Learning with kernels. Technical University of Berlin, Berlin

Sun L (2004) Research on constructing drought monitoring system using remote sensing data of Beijing. China Agricultural University, Beijing

Suykens JAK, Gestel TV, Brabanter JD, Moor BD, Vandewalle J (2002) Least squares support vector machines. World Scientific Publishing, Singapore

Wang XiZhi, Chang JieTian, Wang LiHe Zheng Yi, Ge ShuChun (2012) Change law of soil moisture content under irrigation condition in Henan Province. J Henan Agric Sci 41(2):73-77 